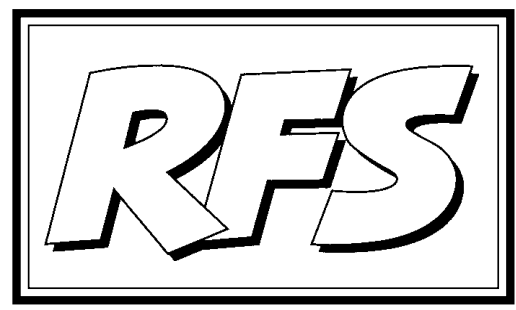

Revista de Fomento Social, 53 (1998), 367-398

\title{
La pequeña y mediana empresa: límites, alcance y posibilidades ${ }^{(*)}$
}

Lapequeña y mediana empresa (PYME) es objeto de numerosas referencias en el mundo socioeconómico. Desencantados del impacto real de la gran empresa existe cierta tendencia a redescubrir el valor de las pequeñas experiencias empresariales. En el presente trabajo se plantean tres objetivos relacionados con este agente empresarial: delimitar el alcance de sus condi-

(*)El presente trabajo constituye una versión más elaborada de una conferenciaimpartidabajo el título ¿Qué es lo que hace triunfar a una PYME? en unas Sesiones de Cultura Empresarial que se desarrollaron en los municipios de Villa del Río, Pozoblanco, y Montilla de la provincia de Córdoba, y organizadas por la Excma. Diputación bajo el amparo de un Proyecto ADAPT financiado por elFondo Social Europeo. Allí tuvimos la oportunidad de conocer, enriquecery confrontar nuestras ideas con empresarios andaluces de éxito comoNúñez de Prado (Aceite), Productos Veleta (Dulces), Torrinco (Carpintería Metálica), Oleícola El Tejar, Almendras Morales (Alimentación) y Plastienvase (Industrial). Posteriormente también hemos finalizadoun trabajo de investigación sobre la PYME titulado Empresas y emprendedores en Córdoba: treinta casos de éxito que también ha nutrido empíricamente estas ideas. Agradezco al Consejo de Redacción de la revista y en especial al Prof. D. Adolfo Rodero Franganillo todas las sugerencias realizadas para la mejora del presente estudio, aunque la responsabilidad de todo lo escrito le corresponde sólo al autor. 
cionamientos íntrínsecos -originados por su tamaño-, clarificar las posibilidades efectivas respecto a las expectativas que despiertan en el mundo económico, y por último, proponer algunas estrategias de acción entresacadas de algunas experiencias empresariales con éxito. En este empeño se pondrán de manifiesto los inconvenientes y ventajas de la PYME respecto a las empresas de otra dimensión. En segundo lugar, se tendrán que deshacer una serie de mitos acerca de la pequeña y mediana empresa que se han idopropagando poco a poco. Este horizonte desmitificado nos conducirá a un perfil más cercano de lo que está ocurriendo en la realidad empresarial, y nos permitirá trazar a grandes rasgos unas líneas básicas en las coordenadas de una pequeña y mediana dimensiónpara un funcionamiento empresarial con éxito.

\section{Alfonso Carlos MORALES GUTIÉRREZ (**)}

Según pone de manifiesto elEstudio Schober PDM(1996) el tejidoempresarial en España (2) se componía de dos millones de unidades de negocio desde grandes empresas a profesionales y pequeños negocios familiares. El volumen de ventas manejado por un $83 \%$ de estas entidades -más de 1.930 .000 unidades empresariales- es de una cuantía inferior a los 100 millones de pesetas. De los casi dos millones de núcleos de actividad, el apartado específico de empresas estaría compuesto por algo más de 790.000 unidades si descontamos las actividades correspondientes a la categoría de profesionales independientes -abogados, médicos, ingenieros o arquitectos-y de pequeños negocios -minoristas, bares, etc.-.

La atomización del tejido empresarial español es un hecho que se mantiene desde hace años pues la inmensa mayoría podría considerarse como pequeñas y medianas empresas. Las grandes empresas son las más famosas pero también son claramente minoritarias en el panorama empresarial español. Sólo el $1 \%$ cuenta con más de cien empleados, mientras que el 58\% de los núcleos empresariales tienen menos de diez trabajadores. Esta situación se mantendrá a medio y

(**)Profesor del Departamento de Economía, Ciencias Jurídicas y Sociología de la Facultad de C. Económicas y Empresariales (ETEA) adscrita a la Universidad de Córdoba.

(2) Citado en Cinco Días, 23 de mayo de 1996 
posiblemente largo plazo. Ante este panorama hablar y profundizar en torno a la PYME representa situarnos en uno de los ejes fundamentales de la actividad económicaespañola.

En algún momento puede desprenderse de las propuestas que se exponen a continuación una postura apriorística hacia este tipo de entidades que podría considerarse como favorable (3). Su posición competitiva de cierta indefensión, su pequeño tamaño que dificulta sus posibilidades y el carácter predominante autóctono podrían ser argumentos, al menos discutibles, que justificarían esta punto de partida que hemos pretendido dejar a un lado en nuestras proposiciones. Dicho esto, el recorrido del presente trabajo ha sido el siguiente: en primer lugar se realiza una delimitación de la PYME destacando sus peculiaridades socioeconómicas; en segundolugar se enumeran ventajas e inconvenientes respecto a otro tipo de realidades empresariales -las grandes empresas-; en tercer lugar se establece un posible marcode evolución y se señalan condicionantes a nivel interno y externo; y por último se proponen algunos factores que pueden permitir que una PYME alcance cotas elevadas de excelencia empresarial.

\section{Delimitación de la PYME}

\section{a) ¿Qué hay detrás de la palabra PYME?}

La expresión PYME alude al conjunto de pequeñas y medianas empresas que se integran dentro de la estructura productiva. La desigual disponibilidad de datos, las diferencias en el grado de desarrollo económicoy la variada intensidad de capital de las distintas actividades económicas impiden que se adopte una definición generalizada. Los parámetros aplicados para encontrar una definición única de PYME varían en función del tratamiento diferencial que se aplique (normas laborales, fiscales, contables, acceso a programas de apoyo financiero...). El límite de lo que es una PYME y lo que no es resulta difuso. Generalmente se utiliza su

(3) Las posturas al respecto son muy diversas. Desde los que apuestan por sus posibilidades (K. CAMPBELL (1997) «El pez chico puede comerse al pez grande» Expansión, 1 de diciembre, p.10; y hasta los que se posicionan con un sentido más crítico(B. HARRISON (1994)El mito de las pequeñas empresas, Harvard Deusto Business Review, nº 5, pp.30-39; B. Harrison (1997) La empresa que viene, Paidós Empresa, nº 46, Barcelona. 
dimensión por volumen de negocio o por número de trabajadores. Se suele tomar los 250 empleados como límite superior(4).Teniendo en cuenta siempre el peligro de parcialidad en que se incurre al acotar un colectivo tan heterogéneo, pueden enumerarse otras características organizativas, más o menos comunes, que pueden ayudar a su catalogación:

1. En cuanto a la propiedad: son empresas independientes, gestión y propiedad suelen estar unidas en la misma persona. Esta particulariedad se encuentra intimamente relacionada con el carácter familiar de estas experiencias.

2. En cuanto a su estructura interna: su estructura organizativa es simple (5), es decir, una departamentalización funcional rudimentaria, escasa burocratización, centralización de decisiones, etc.

3. En cuanto al entorno en el que se desenvuelven: carecen de posición dominante en el mercado. Así en El Informe Notemboom, aprobado por el Parlamento Europeo en 1978, se afirmaba: «las pequeñas y medianas empresas son indispensables para mantener el orden económico en la medida en que contribuyen a afrontar riesgos, responsabilidades y propiedad y, lo que es más importante, a mantener la competencia. Las posiciones de dominio apenas pueden implantarse alli donde existen pequeñas y medianas empresas bien concebidas y bien dirigidas».

La dificultad para asignar límites precisos a este colectivo no impide reconocer que su existencia tiene unos efectos económicos y sociales de creciente interés (6).

(4)La Unión Europea presentó un proyecto de recomendación en 1996 en el que consideraba tres niveles de PYMEen función del número de empleados, volumen de negocio en ecus, balance total, y que no esté participada en más de un $25 \%$ por empresas de mayor dimensión. Estos niveles serían: mediana empresa (menos de 250 empleados, menos de 40 millones de ecus de facturación, menos de 27 millones de ecus de balance total), pequeña (menos de 50 empleados, menos de 7 millones de ecus de facturación y menos de 5 millones de ecus de balance total) y microempresa (menos de diez trabajadores).

(5)En términos organizativos se denomina configuración emprendedora o simple. Un análisis más detallado de este tipo de características puede verse en A.C. Morales GutiérRez (1997).

(6) Según el estudio titulado Las empresas españolas en las fuentes tributarias, que ha realizado el Instituto de Estudios Fiscales (IEF) con información referida a 1990, la participación en la economía española de las PYME-considerando como tales a la empresas que tiene menos de 250 trabajadores y teniendo en cuenta que las diferentes necesidades de tecnología y capital determinan una distribución sectorial muy diferenciada- se refleja en los datos siguientes: representan el 99,83\% de las empresas y a ellas corresponden el 67,33\% de los empleados, el 
A título indicativo puede decirse que las empresas con menos de 10 trabajadores en España ocupan un $43 \%$ del empleo y realizan un 35\% de las ventas, frente al $32 \%$ y $20 \%$ respectivamente en la Unión Europea.

\section{b) Detrás de una PYME hay un emprendedor o una emprendedora}

La vinculación entre la empresa y su propietario es muy estrecha en el caso de la PYME. La figura de empresa-empresario-directivo se concentra e identifica generalmente en una sola persona: el emprendedor. Por ello para conocer el funcionamiento y el dinamismo de estas entidades puede resultar de interés conocer el perfil y las actitudes de las personas que representan un papel protagonista deestas iniciativas. La Encuesta de Población Activa facilitaba(CES, 1995) algunos datos sobre las características de los empresarios españoles: el $74,2 \%$ son varones, sólo el $16 \%$ son técnicos profesionales o universitarios y el $50,7 \%$ tienen una edad comprendida entre los 30 y 49 años. La presencia de las mujeres en la actividad empresarial así como el número de empresarios más jóvenes con mayor nivel de formación y mayor tendencia a ejercer su actividad en equipo va en aumento (7).

Este perfil básico del emprendedor se completa con una serie de actitudes personales más o menos generalizadas:

- necesidad de independencia en grado más elevado que el resto de la población en general,

- acceso temprano al trabajo, ya sea en el negocio familiar o fuera de él,

- asunción de la responsabilidad por el resultado de las propias acciones,

- alta capacidad de iniciativa y deseo de éxito,

- propensión a asumir riesgos, pero moderados y en la medida en que pueda utilizar su juicio e ingenio para influir en el resultado de sus acciones.

Estas características no son ajenas al empresario español y están estrechamente

64,25\% de las ventas, el 49,59\% de los salarios, el 41,05\% de las importaciones y el 56,33\% de la recaudación por IVA.

(7) Según un informe de la Escuela de Organización Industrial (EOI) la mitad de las empresas que se han creado en España durante 1997 están dirigidas por mujeres. El dato es sorprendente si se compara con el 16\% de emprendedoras en el año 1990 (Expansión, 20 noviembre de 1997, p.16). 
ligadas al «controlinterno», o creencia de que la persona puede ejercer algún dominio sobre los hechos y el entorno. Esta actitud se contrapone al «control externo», o creencia en que los acontecimientos están controlados por fuerzas ajenas.

Una vez situado el contex to organizativo y humano de la PYME pasamos a delimitar las restricciones y ventajas que implica desarrollar la actividad económica en una unidad empresarial en el marco de estas características.

\section{Alcance y posibilidades de la PYME}

\section{a) Lo pequeño es hermoso y lo grande poderoso}

La teoría de las organizaciones propugna una serie de hipótesis respecto al tamaño organizativo que han sido constatadas empíricamente. Por ejemplo a mayor tamaño mayor burocratización. Sin embargo las ventajas que comporta un gran tamaño, tambien pueden constatarse. Diversos estudios nos muestran, entre otras, las siguientes tendencias a nivel de proceso funcional (8):

1. Función comercial: Los recursos dedicados a variables fundamantales del marketing -gastos de publicidad sobre ventas-, aumentan a medida que se incrementa el tamaño de las empresas.

2. Función tecnológica: La actividad tecnológica-porcentaje de gastos en I+D sobre las ventas y del personal dedicado a I+D sobre el empleo total-también crece con el tamaño (9). Sin embargo, cuando las PYME se deciden a utilizar las nuevas tecnologías, lo hacen en mayor proporción y su esfuerzo investigador resulta superior al de las grandes empresas (10).

(8) En este caso nos referimos a la Encuesta sobre Estrategias Empresariales (ESEE) realizada por el Ministerio de Industria y aplicada a una muestra representativa de 2.198 empresas industriales durante 1990. A pesar de su desfase temporal su validez resulta indiscutible, puesto que se refiere a comportamientos que permanecen y que viene condicionados por el tamaño de las empresas.

(9) Un porcentaje muy reducido de las empresas más pequeñas utilizan nuevas tecnologías aplicadas a la producción, tales como elCAD (diseño, asistido por ordenador), la robótica o los sistemas flexibles. Un 15,3\% trabajan con máquinas-herramientas de control numérico. No obstante el grado de utilización de nuevas técnicas varía de unos sectores a otros.

(10) En cualquier caso todas las empresas españolas son muy dependientes del exterior en este terreno. Son las PYME de 51 a 100 trabajadores que acometen actividades de I+D las empresas 
3. Función laboral: La PYME muestra una mayor propensión a los contratos eventuales pero la duración media de estos contratos es mayor (11). Además cuanto menor es la empresa mayor es el número de horas trabajadas al año por persona ocupada. Con las horas extraordinarias y con las horas no trabajadas el comportamiento es inverso.

4. Función inversora: La intensidad inversora es correlativa con el tamaño empresarial. La inversión por trabajador pasa de 395.000 pesetas en las empresas más pequeñas a 1,2 millones en las de más de 500 trabajadores. No obstante la variable sectorial resulta determinante a la hora de confirmar esta tendencia (12).

5. Dimensión internacional: La propensión a exportar(13) o importar-relación entre el valor de estas operaciones y el volumen total de ventas- no está condicionada por el tamaño de la empresa. Pero el tamaño de la empresa resulta un claro condicionante para la atracción de capital extranjero (14).

6. Función de recursos humanos: La posibilidad de invertir y el porcentaje de recursos que se destinan a formación crece con el tamaño de la empresa (15).

7. Función financiera: La debilidad intrínseca de las PYME en cuanto a sus

que aplican en mayor proporción tecnología nacional.

(11) Las PYME suscriben el 80\% de la contratación temporal (Capital Humano, $\mathrm{n}^{\circ}$ 104, octubre 1997,p.6). En el mismo sentido apuntan informaciones comola Encuesta de Coyuntura Laboral, aunque los tramos de empresa representados no sean homogéneos.

(12) Hay sectores en los que la proporción de valor añadido que se destina a la compra de bienes de equipo es superior en las PYME con respecto a las grandes empresas, aunque no lo sea la inversión por trabajador como ocurre en los sectores de productos alimenticios, textiles y calzados. La cantidad invertida por trabajador en los distintos sectores es tanto menor cuanto mayor es su intensidad de mano de obra.

(13) La propensión exportadora es más elevada en los sectores de «máquinas de oficina» (18\%), «vehículos automóviles y motores» $(19,5 \%)$ y «cuero y calzado» $(19,1 \%)$.

(14) E1 92,6\% de las empresas con menos de 200 trabajadores sólo tienen participación nacional, mientras que en el caso de las empresas con más de 200 trabajadores este porcentaje cae al 52,9\%. Bien es cierto que, cuando se produce la toma de participación por parte del capital extranjero, ésta es mayoritaria y, por tanto controla las decisiones de la empresa. Este caso se da en el 64\% de las PYME y en el 82\% de las grandes empresas participadas.

(15) Es significativo que el 79,7\% de las empresas encuestadas con menos de 200 trabajadores realizan alguna inversión en formación, frente al 97,3\% de las empresas de más de 500. 
posibilidades de obtención de recursos financieros se fundamenta en unos recursos propios siempre restringidos. Esta situación les impide además el acceso a fuentes financieras de mayor plazo y menor coste (16).

Por tanto, si las variables estratégicas - como son el marketing, la tecnología o la capacidad para atraer capital-se sitúan de forma más favorable para la empresa grande que para la mediana y pequeña. La conclusión podría ser que lo grande es sinónimo de éxito, mientras que ser pequeño es estar condenado a una falta de competitividad crónica.

Sin embargo las PYME pueden adoptar estrategias más flexibles que las grandes empresas, debido al tipo de contratación en función de las necesidades de la demanda, la menor frecuencia con la que recurren a los contratos de muy corto plazo, el menor número de horas extraordinarias y de horas no trabajadas, las jornadas más largas y salarios más bajos, la menor conflictividad laboral.

En cualquier caso, predomina la idea de que el tamaño es una condición para el éxito, o de otra forma, que la PYME es una empresa infradesarrollada. Paralelamente nos encontramos en una época de euforia respecto al alcance de las PYME. La lógica de las tendencias, el deseo de una realidad menos determinante que se transforma en mito y el análisis de los resultados que reflejan ciertos estudios pueden aportarnos cierta luz ante tal tesitura.

(16) Los datos de la Central de Balances del Banco de España para el bienio 1990-91 de una muestra de 2.683 empresas industriales no energéticas, de las cuales 2.613 eran privadas nos aportan alguna luz sobre el problema a pesar del sesgo hacia las grandes empresas que supone esta fuente. Se partió de una clasificación por tamaño que consideraba que el $68 \%$ eran pequeñas empresas ( $<100$ trabajadores), el $23 \%$ medianas empresas $(>100 \mathrm{y}<500$ trabajadores) y el $9 \%$ grandes empresas ( $>500$ trabajadores). Las cifras de equilibrio financiero de estos tres grupos confirman que el peso de la estructura fija respecto al activo neto es mayor en las grandes empresas $(67,21 \%$ que en las medianas $(57,87 \%)$ y en las pequeñas $(43,79 \%)$. Estas cifras resultan coherentes con la mejor tasa inversora ya comentada y están en función, principalmente, del mayor porcentaje que representan en las grandes empresas los recursos ajenos a largo plazo sobre el pasivo remunerado: un $28,03 \%$ frente al $15,01 \%$ en las pequeñas. Los recursos propios sobre pasivo son, por el contrario, más gravosos en las pequeñas y medianas que en las grandes empresas, loque refleja la mayor dependencia de la financiación propia en las empresas de menor tamañoy, por tanto, su estrecha vinculación con la coyuntura económica. También en este sentido opera la mayor proporción de la financiación a corto plazo sobre el pasivo remunerado en las pequeñas empresas $(25,60 \%)$ que en las grandes $(22,44 \%)$. Destaca, al mismo tiempo, su mayor dependencia del descuento de efectos: el 8,96\% en las pequeñas, frente al 3,36\% en las grandes. 


\section{b) Mitos y realidades respecto a las PYME}

Para desmitificar la idea «PYME» tenemos que fijarnos en qué empresas son las que realmente están creando empleo en Europa, quiénes son los empresarios que están creándolas, cuál es el perfil de estos empresarios de éxito y qué estrategias siguen. La Fundación Europea para la Investigación de la Iniciativa Empresarial (17) realizó un estudio con el auspicio de la Comisión Europea sobre Los 500 empresarios más dinámicos de Europa: los creadores de empleo. Dicha investigación ha venido a confirmar muchas de las ideas más extendidas sobre la iniciativa empresarial en Europa, pero también hallevado a poner en tela de juicio algunos de los mitos más comunes sobre la creación de empleo y las empresas de éxito con altas tasas de crecimiento (18). Dadoel interés de lainvestigación-puesto que algunas de sus conclusiones se refieren a las PYME- a continuación destacaremos las aportaciones más relevantes (Roure, 1996):

1) A veces se afirma que sólo las PYME son las que más empleos crean, sin embargo la realidad demuestra que sólo un determinado tipo de PYME-las empresas y empresarios dinámicos-son las que registran altas tasas de crecimiento y generan empleo (19).

2) Muchas veces se escucha que la principal fuente de crecimiento del empleo son las empresas de nueva creación (20). Pero la generación de empleo

(17) European Foundation for Entrepreneurship Research (EFER)

(18)La muestras de la investigación se limitó a empresas de tamaño medio con menos de 500 empleados en 1989 (al inicio del período estudiado) y más de 40 en 1994 (el último año estudiado). En todas las empresas, el empresario (o empresarios) poseían una proporción importante de capital.

(19) En el período estudiado, el número de puestos de trabajo existentes en el conjunto de Europa, incluidos todos los tipos de empresas, se redujo en casi dos millones en términos netos $\mathrm{y}$, aunque la mayor parte de estas pérdidas son atribuibles a las grandes empresas, en torno a 700.000 puestos de trabajo perdidos corresponden a las PYME. En cambio, la muestra de 500 empresas que identificó el estudio citado (con un crecimiento anual medio en torno al $21 \%$ en términos de empleo y del $29 \%$ en términos de venta) consiguió crear más de 100.000 empleos.

(20) Algunas encuestas estiman en 3 años la vida media para el $50 \%$ de las nuevas empresas. Al mismo tiempo, asistimos a un creciente protagonismo de las unidades con menos de 5 trabajadores. Al final del período de 2 años comentado se había creado en España una nueva microempresa por cada dos mil habitantes, pero se habían perdido 33.000 empresas medianas y grandes. 
puede constatarse por igual tanto en empresas maduras (21) como en aquellas de nueva creación.

3) Podría pensarse que las empresas con altas tasas de crecimiento se concentran en sectores nuevos. Sin embargo las empresas dinámicas pueden encontrarse en todos los sectores (22).

4) El estereotipo del empresario de una entidad con alta tasa de crecimiento es el de una persona joven y muy cualificada. Sin embargo, los empresarios dinámicos son de todas las edades y niveles educativos (23).

5) Existe el mito de que las empresas con altas tasas de crecimiento tienen detrás a un empresario enérgico y autosuficiente. Sin embargo las empresas dinámicas tiene detrás a equipos con un enfoque profesional de la dirección de empresas (24).

6) Podría formularse que las empresas con altas tasas de crecimiento se orientan hacia mercados en crecimiento. Sin embargo las empresas dinámicas se orientan hacia segmentos del mercado en los que pueden ser los líderes o al menos un contrincante importante (25).

(21) El colectivo de 500 empresas seleccionadas llevan operando una media de 17 años. De hecho, cuatro de ellas se crearon inicialmente en el siglo XIX, y sóloel 14\% tenía menos de 5 años en 1994.

(22) La distribución sectorial de las 500 empresas más dinámicas de Europa es bastante similar a la del conjunto de las PYME. Con todo, el sector servicios en su conjunto parece proporcionar un terreno más abonado parra el crecimiento que la industria manufacturera. Este resultado parece demostrar que no existen sectores de éxito, sino, más bien, empresarios y organizaciones de éxito.

(23) Tres cuartas partes de los empresarios más dinámicos de Europa tienen edades comprendidas entre los 35 y los 65 años. Sólo el $7 \%$ tienen menos de 34 años y el 18 por ciento más de 55 años. Los empresarios dinámicos presentan unos antecedentes educativos muy variados, y menos de una tercera parte de ellos tienen un título universitario.

(24)La mayoría de los empresarios estudiados parecen tener una gran confianza en su propia capacidad para identificar y aprovechar oportunidades, superando toda clase de obstáculos. En cambio, son plenamente conscientes de los retos que supone sacar adelante una empresa en el entorno competitivo actual y, por lo tanto, forman equipos para complementarse en la dirección de la empresa.

(25) Más del $80 \%$ de los 500 empresarios más dinámicos de Europa se consideran un agente relevante en su segmento de mercado. Estas empresas tratan de evitar la competencia concentrándose en segmentos o nichos que a veces crean ellas mismas, y en los que intentan desempeñar una función importante e influyente aplicando estrategias proactivas. 
7) Las empresas con altas tasas de crecimiento se corresponderían con aquellas que se dirigen hacia mercados nacionales en los que pueden desarrollar una posición dominante. Sin embargo las empresas dinámicas destinan una proporción significativas de sus ventas hacia los mercados de exportación (26) en los que pueden aprender y crecer.

8) Las empresas con altas tasas de crecimiento en lugar de aplicar estrategias competitivas de bajos costes compiten con productos y servicios de alta calidad (27).

9) Frente a laidea de que las empresas de éxito utilizan fundamentalmente una tecnología original, la realidad muestra que las empresas dinámicas confían sobre todo en sus empleados, poniendo sumo cuidado en su selección, formación y desarrollo.

10) Podría plantearse como hipótesis que las empresas con altas tasas de crecimiento utilizan fuentes de financiación muy complejas para su desarrollo. Sin embargo las empresas dinámicas se financian fundamentalmente con sus recursos propios y con la ayuda de créditos bancarios (28).

Podría formularse como síntesis de la desmitificación realizada que una PYME no es una empresa de éxito por su tamaño, ni su tamaño impide que necesariamente

(26) En torno al $80 \%$ de las 500 empresas más dinámicas de Europa tienen una presencia activa en los mercados de exportación. Como media, la parte de las exportaciones es del $44 \%$ de sus ventas, y las exportaciones se dirigen sobre todo a otros Estados miembros de la Unión Europea. Las empresas dinámicas exportan un porcentaje mucho mayor de sus ventas que las PYME europeas en general, utilizan fundamentalmente canales de distribución directos y, en mucha menor medida, inversiones directas en el extranjero, empresas conjuntas o licencias.

(27) Los empresarios más dinámicos de Europa han optado por estrategias de diferenciación -en calidad de sus productos y servicios-con mucha mayor frecuencia que por estrategias de bajos costes. Estas estrategias se sustentan en una elevada participación de los empleados y clientes en el proceso de mejora.

(28) Los empresarios más dinámicos de Europa se esfuerzan por mantener su independencia financiera en la medida de los posible. En el $87 \%$ de las empresas, el empresario principal controla más de la mitad de las acciones. La fuente de financiación más importante de este tipo de empresas son los beneficios no distribuidos. Los créditos bancarios son la única fuente de financiación significativa además de aquélla. El capital de riesgo y los inversores privados sólo revisten cierta importancia en el $10 \%$ de las empresas que han salido a cotización en bolsa. Sin embargo, otro $30 \%$ está considerando salir a cotización, lo que puede llevarles a dar una mayor importancia al capital de desarrollo. 
tenga éxito. Para completar nuestro análisis podría ser interesante poner de manifiesto las etapas de desarrollo de una empresa para situar que muchas PYME fracasan precisamente porque no superan determinadas etapas en las que el factor tamaño no resulta decisivo, otras empresas evolucionan cualitativamente más que cuantitativamente y se sitúan en una etapa determinada, y otras evolucionan y crecen en tamaño pero se encaminan hacia su ocaso.

\section{El desarrollo de la PYME: evolución y condicionamientos}

\section{a) ¿Es la PYME una empresa infradesarrollada?}

La comprensión de las distintas fases de desarrollo de una empresa puede ayudarnos a acotar esta proposición. Además, de la misma forma que muchas de las características básicas de nuestra personalidad se forjan en los primeros años de la infancia, muchas de las virtudes y defectos de la pequeña y mediana empresa están presentes o se generan en sus primeros momentos de vida. Los empresarios capaces de situarse y determinar en qué fase de desarrollo empresarial se encuentran pueden usar el marco de análisis que se facilita a continuación para comprender mejor los problemas que afrontan y anticiparse a los retos que pueden avecinarse. Dicho marco (Churchill, 1996) comprende seis fases de desarrollo, cada una caracterizada por un índice creciente de tamaño, complejidad y/o diversificación, y definida por cinco factores: estilo de dirección; estructura organizativa; desarrollo de sistemas formales; grandes objetivos estratégicos; e implicación del propietario en la empresa. Seguidamente describimos los rasgos más característicos de cada una de estas etapas aplicables tanto a la pyme-sobre todoen las primeras etapas-como a la evolución de grandes empresas-reflejada en las últimas-:

- Fase I: Nacimiento. Se caracteriza por la existencia de una serie de problemas básicos no resueltos: los clientes son insuficientes, o no se puede suministrar el

(29) Se pueden proponer como cuestiones claves en esta fase las siguientes: ¿Podemos conseguir suficientes clientes y suministrar nuestros productos con un nivel de calidad suficiente para convertirnos en una empresa viable? ¿Podemos expandirnos a partir de un cliente clave, accediendo a una clientela más amplia? ¿Podemos desarrollar el producto desde el proceso de producción piloto a un proceso de producción estandarizado? ¿Tenemos suficiente dinero para atender las ingentes necesidades de liquidez a los inicios? 
producto o servicio contratado en la cantidad necesaria y con la calidad requerida (30). Las organización es muy simple; el propietario es la empresa, realiza todas las tareas importantes y aporta la mayor parte de la energía, la dirección y, junto con sus parientes y amigos, inclusoel capital. Los sistemas y la planificación formal o son mínimos o inexistentes. La estrategia de la empresa consiste en sobrevivir. Muchas de estas experiencias nunca llegan a conseguir un número suficiente de clientes como para ser viables, el capital se va agotando y los propietarios terminan por liquidar el negocio. En otros casos, los propietarios no consiguen asimilar las exigencias que les plantea la empresa en términos de tiempo, dinero y energía y tiran la toalla. Las que consiguen mantenerse a flote pasan a la fase siguiente.

- Fase II: Supervivencia. La empresa ha demostrado su viabilidad: cuenta con un clientela suficiente y tiene capacidad para conservarla. El problema fundamental pasa a ser la relación entre los ingresos y los gastos. La organización sigue siendo muy simple, posiblemente con un número limitado de empleados supervisados por un director de ventas o un encargado general. Ninguno de ellos adopta las decisiones importantes por sí solo, sino que se limitan a aplicar estrictamente las instrucciones del propietario. La planificación formal se limita por lo general a la elaboración de previsiones financieras y a los planes de negocio. La supervivencia sigue siendo el objetivo principal. Durante esta fase, la empresa puede crecer en tamaño y en rentabilidad y pasar a la fase siguiente, $\mathrm{o}-$ comoles sucede a muchasestancarse durante mucho tiempo (31).

(30)Las principales cuestiones de esta fase serían: ¿podemos generar suficiente liquideza corto plazo como para alcanzar el equilibrio financiero y financiar el mantenimiento o la sustitución de nuestros activos de capital a medida que se desgasten? ¿Podemos generar suficiente liquidez, como mínimo, para mantenernos en el negocio y financiar el crecimiento hasta alcanzarun tamaño lo suficientemente grande como para obtener un beneficio económico con nuestros activos y nuestropersonal?

(31) Muchas de estas empresas se crean a partir de un hobby del propietario, y en donde los aspectos no económicos de la empresa, desempeñan un papel positivo. También pueden ser empresas familiares, como por ejemplo pequeños restaurantes. En muchos casos, estas empresas consiguen sermuy rentables y pasan a la siguiente fase. En otros, los fundadores eligieronempresas inviables en un sector ya muy congestionado, o se localizaron en un mal emplazamiento, y no consiguen, o no están dispuestos, a hacer todo lo necesario para conseguir que sean rentables. Explotan este tipo de negocio hasta que mueren o se jubilan, y aunque algunos de ellos se venden o traspasan a hijos que desean reformarlos, la mayoría simplemente dejan de funcionar y desaparecen. 
- Fase III: Estabilización. La empresa ha alcanzado un estado saludable: tamaño suficiente, posición cómoda en el mercado y obtención de una rentabilidad superior a la media. La empresa puede mantenerse en esta fase de manera indefinida siempre y cuando no se produzcan cambios inesperados del entorno o una gestión deficiente reduzca su competitividad. Desde el punto de vista organizativo, la empresa ha crecido lo suficiente como para necesitar directivos funcionales que asuman algunas tareas especializadas lo que propicia el que la identificación entre la empresa y su propietario vaya diluyéndose. La liquidez abunda, y la principal preocupación reside en evitar el despilfarro en los períodos de mayor prosperidad. Se implantan sistemas básicos de gestión financiera, marketing, producción y una planificación articulada en torno a los presupuestos operativos. Muchas empresas se mantienen durante mucho tiempo en esta fase (32).

-Fase IV: Crecimiento. El propietario-directivo consolida la empresa utilizando la liquidez y lacredibilidad consolidada para financiar su crecimiento. Las tareas más importantes consisten, además de asegurar la rentabilidad y la liquidez, en contratar y desarrollar aquellos directivos de mayor potencial dado que de ellos depende el futuro de la organización. El propietario delega la planificación operativa y comparte con los directivos claves la planificación estratégica, pero sigue como propietario/directivo partícipe en todas las áreas de actividad de la empresa. Si tiene éxito, la empresa puede crecer rápidamente y evolucionar a la siguiente fase (33).

- Fase V: Despegue. En este momento el problema clave es cómo conseguir

(32) En algunos casos, el nicho de mercado que ocupan sus productos no les permite crecer, como sucede con muchas empresas de servicios en localidades pequeñas y con los titulares de franquicias sujetos a límites territoriales. Si la empresa consigue adaptarse de manera continuada a los cambios del entorno, puede seguir así de manera indefinida, o ser vendida o fusionada de manera ventajosa, otambién seguir creciendo. En el caso de los titulares de franquicias, estaúltima opción requeriría la adquisición de nuevas franquicias. Si la empresa no consigue adaptarse a las circunstancias, como les sucedió a muchos concesionarios de automóviles a finales de los años setenta y primeros ochenta, seguramente retrocedería hacia la fase de supervivencia.

(33) De hecho, la Fase IV suele ser un primer intento de crecer, por ejemplo a nivel regional, antes de lanzarse por entero por la senda del crecimiento. Si una empresa que se encuentre en la Fase IV fracasa, puede detectar las causas a tiempo para retrotraerse a la etapa anterior. De lo contrario, tal vez sea posible empezar de nuevo en la Fase II antes que quiebre o se vea obligada a una venta apresurada. 
y financiar un crecimiento rápido. Las cuestiones más relevantes que se derivan de tal decisión son: la delegación (34), la gestión de tesorería (35) y el control de costes. La organización es descentralizada y, al menos en parte, se estructura en departamentos de naturaleza funcional. Los directivos claves deben mostrar un grado de competencia suficiente como para manejar la complejidad y el dinamismo que supone esta situación. Los sistemas adquieren mayor sofisticación. La planificación se convierte en una actividad permanente, y en ella participan todos los directivos claves. Aunque el personal de dirección ha aumentado considerablemente, la empresa sigue dominada aún por la presencia de propietario/directivo y por el control de la propiedad. Este es un período central en la vida de la empresa. Si el propietario supera los desafíos, tanto financieros como de gestión, de una empresa en crecimiento, puede convertirse en una gran empresa. En caso contrario existe la posibilidad de venderla -de forma ventajosa-, siempre y cuando el propietario reconozca a tiempo sus limitaciones (36). Si una empresa no consigue dar el gran salto adelante, tal vez pueda mantenerse siendo una empresa de éxito en estado de equilibrio con un tamaño adecuado o retroceder si los problemas son demasiado profundos.

- Fase VI: Madurez. Consolidar y controlar los beneficios financieros producidos por el rápido crecimiento experimentado y conservar las ventajas que otorga un tamaño pequeño -flexibilidad de respuesta, espíritu emprendedorconstituyen las principales preocupaciones en esta fase. La empresa debe ampliar la composición del equipo directivo lo bastante deprisa como para eliminar las ineficiencias del crecimiento y profesionalizar la empresa con herramientas tales como presupuestos, dirección por objetivos y los sistemas de costes estándar, y

(34) Podrían plantearse como cuestiones importantes en esta faceta: ¿Es capaz el propietario de delegar responsabilidades para mejorar la eficiencia en la gestión de una empresa cada vez más compleja? ¿Se tratará de una auténtica delegación, acompañada de controles del rendimiento y buena disposición para detectar los errores, o más bien de una auténtica abdicación?

(35) Son cuestiones clave a este respecto: ¿Se generará suficiente liquidez a partir de las operaciones y los ahorros de la empresa como para satisfacer las enormes exigencias que el crecimiento impone? De no ser así, ¿dónde y cómo puede conseguir financiación adicional? ¿Tolerará el propietario un alto coeficiente de endeudamiento o la dilución de su capital?

(36) Esta situación se produce frecuentemente, ya sea porque intentan crecer demasiado deprisa y se quedan sin dinero (el propietario cae víctima del síndrome de omnipotencia) o porque son incapaces de delegar (el síndrome de la omnisciencia). 
hacerlo sin ahogar su espíritu emprendedor. Se dispone del personal y los recursos financieros necesarios para proceder a una detallada planificación tanto operativa como estratégica. La dirección está descentralizada y cuenta con una dotación suficiente de directivos con la experiencia adecuada. El propietario y la empresa son dos cosas muy distintas, tanto financiera como operativamente. Pero la empresa no hallegado a la meta. Cuenta con las ventajas que le aporta su tamaño, sus recursos financieros y su talento directivo. Si consigue mantener su espíritu emprendedor, tendrá una vitalidad extraordinaria, en caso contrario puede encontrarse en lo que puede considerarse como una séptima etapa: la osificación.

- Fase VII: Osificación. Se caracteriza por la falta de decisiones innovadoras y la aversión a todo tipo de riesgos. Esta etapa parece ser más común en las grandes corporaciones cuyas cuotas de mercado considerables, poder de compra y recursos financieros las hacen viables hasta que se produce un cambio significativo en el entorno. Desgraciadamente, suelen ser los competidores -que crecen más rápido-los que advierten estos cambios con mayor antelación.

\section{b) Condicionamientos internos: dificultades para crecer y perdurar}

La evolución probable de una entidad nos muestra diversos estados de crecimiento y las características que lo acompañan. Dado que el objeto de nuestro estudio son las PYME puede ser de interés el reflexionar sobre aquellas cuestiones críticas que pueden posibilitar o restringir esa pérdida de identidad por su dimensión: convertirse en una gran empresa. Hemos elegido dos cuestiones al respecto que abordaremos sucintamente: las dificultades para el crecimiento y los problemas de la sucesión de los fundadores.

\section{b.1) Una decisión crítica: dejar de ser una PYME}

Crecer es un concepto inherente a la dinámica empresarial. En el desarrollo de la PYME puede surgir la decisión, o la oportunidad, de dar un salto cuantitativo y cualitativo que consecuentemente lleva aparejado el dejar de ser una PYME. Dicha decisión surge concatenada con otras. Por un lado, es posible que la empresa necesite nueva financiación externa para posibilitar su expansión. Por otro lado, el aumento del tamaño de la organización puede obligar al empresario a cambiar la manera en que se gestiona la empresa, al reducirse considerablemente el control 
directo de sus operaciones cotidianas.

Una de las consecuencias más evidentes ante el crecimiento consiste en la pérdida de cercanía y de calor humano a nivel de la cultura organizativa a medida que la empresa crece (Levié, Hay 1996). Los empleados que llevan en la empresa desde los primeros momentos se quejan de que las relaciones con el empresario ya no son lo que eran. Este problemas tiene su origen en la incapacidad de comunicar los cambios que están produciéndose en la organización, así como en la función del empresario. A menudo, un crecimiento rápido obliga al empresario a dedicar más tiempo a gestionar los recursos externos, como grandes clientes extranjeros, bancos de inversión, accionistas institucionales, medios de comunicación y mercados de valores. Todo esto suele absorber a los empresarios más tiempo -fuera de la empresa- del que esperaban y sus empleados perciben que ya no está tan disponible como antes (37).

El temor a la pérdida del control respecto a otras personas del ámbito interno de la organización es innato al empresario. Pero mucho más explícito es su temor a perder control frente a los proveedores en general, y a las entidades bancarias, en particular. La incapacidad de establecer y mantener con los proveedores de recursos una relación estrecha basada en la confianza y el beneficio recíproco puede traducirse en contratos jurídicamente muy complejos, la exigencia permanente de informaciones relativamente triviales pero que consumen una gran cantidad de tiempo y la retirada inesperada de los recursos precisamente en el momento que más se necesitan. No todo es culpa del empresario. Por supuesto, algunos proveedores de recursos pueden no responder a la necesidad de una empresa que comienza de establecer relaciones a largo plazo basadas en la confianza y el beneficio mutuo. Al fin y al cabo, los proveedores de recursos deberían actuar como agentes del cambio, no como barreras al crecimiento.

Si un empresario decide no asumir la decisión de crecer sus estrategias de acción se reducen considerablemente. Lo normal sería coexistir con estas barreras e identificar y mantener un pequeño nicho de mercado protegido y

(37) Una solución estructural, a la que han recurrido con éxito muchas organizaciones empresariales de éxito, consiste en replicar, en lugar de sustituir, la vieja cultura informal de los comienzos. Una manera de hacerlo es superponer a la incipiente estructura funcional un mosaico de equipos de proyecto multifuncionales. Los empresarios más avispados incorporan como miembros de estos equipos a asesores, clientes y proveedores cuando es oportuno. Estos equipos se forman para responder a necesidades específicas y se disuelven al concluir su misión. 
practicar la autosuficiencia financiera y de gestión. Sin embargo, cuando no existen grandes barreras de entrada en el mercado, o existe cierta inestabilidad tecnológica, la viabilidad de una estrategia de este tipo disminuye. Si la dirección de la empresa es incapaz o no está dispuesta a superar mediante el crecimiento su actual situación de debilidad estratégica, la competencia se intensificará a medida que competidores más capaces -0 , simplemente, con más recursos- entran en sus dominios.

Existen dos tipos de barreras: las externas y las internas. Según diversos estudios (38) las dos principales barreras internas a la hora de plantearse un mayor desarrollo empresarial son la capacidad de la dirección para manejar el crecimiento y las reticencias de los propietarios/directivos a ceder parte de su control a cambio de financiación para el crecimiento (en forma de recursos propios o ajenos). En cuanto a las barreras externas las citadas con mayor frecuencia son la existencia de un mercado caracterizado por una fuerte competencia y un escaso potencial de crecimiento. A las segundas se atribuye por parte de los empresarios mayor importancia que a las primeras.

Cabría preguntarse si los temores que aparecen como barreras internas son reales o infundados. Respecto a la convicción de que, para obtener los recursos necesarios para el crecimiento de la organización y, por consiguiente, para su supervivencia, el empresario no tiene más remedio que ceder el control de la empresa-accionistas, bancos,...-(barrera de pérdida de control) conviene poner de manifiesto su inconsistencia con una visión moderna de la empresa. Al plantearse el crecimiento en términos de control-y no de uso (39)-de los activos, el empresario aplica modelos anticuados de capitalismo que equiparan la propiedad con el control, así como modelos anticuados de pensamiento en la gestión que consideran el control como la única forma de dirección posible. Esta diferencia conceptual no gira sólo en torno a la alternativa entre alquilar o comprar. Se refiere

(38) Según las encuestas llevadas a cabos en el marco de las investigaciones de la London Business School, en las que se comparan los resultados de las encuestas realizadas entre pequeñas y medianas empresas no cotizadas de todo el Reino Unido en 1992, 1994 y 1996 (Churchill, 1996)

(39) Investigaciones realizadas en Estados Unidos han puesto de manifiesto que las empresas de alto crecimiento hacen uso de recursos externos en mayor medida que las empresas de bajo crecimiento. En otras palabras, la prioridad de la dirección de las empresas de alto crecimiento no es tanto el control o la propiedad como el uso de los activos. 
fundamentalmente a la forma de gestionar los activos.

Además el control no sólo consiste en ejercer un férreo poder sobre todos los recursos incluso los humanos. Recientes investigaciones realizadas en Estados Unidos revelan que los máximos responsables de las empresas de mayor crecimiento hacen mayor hincapié en el desarrollo de una cultura corporativa que motive a los empleados y la creación y comunicación de una visión del futuro de la organización que se trasmite no sólo dentro de la empresa sino también a los propietarios de los recursos externos (40).

\section{b.2) Segunda decisión crítica de la PYME: perdurar (41)}

La opción de crecer tiene repercusiones inmediatas y a largo plazo. Puede plantearse que crecer es sinónimo de perdurar pero la realidad no refrenda esta proposición. Perdurar requiere otro planteamiento distinto. En el caso de las PYME su viabilidad a lo largo del tiempo no sólo depende de factores de mercado sino también de factores idiosincráticos. Ya comentábamos la vinculación entre empresario y familia. La problemática de la PYME se encuentra estrechamente asociada a la problemática de la empresa familiar que necesariamente no tiene por qué ser pequeña (42). La impronta familiar condiciona a las PYME en su

(40) No obstante, estos trabajos de investigación han puesto de manifiesto que, durante las fases de rápido crecimiento, estos comportamientos claves suelen verse sometidos a grandes presiones, y la rentabilidad desciende. Una de las principales causas puede ser simplemente que los presidentes se encontraban demasiado ocupados apagando incendios a nivel operativo o inmersos en los nuevos desafíos que el crecimiento entraña (como comunicarse con los nuevos grupos de intereses dentro de la empresa) como para desempeñar la capital función de motivación y comunicación frente a la organización y al resto de los proveedores de recursos.

(41) Algunos de los datos que se vierten en este apartado se refieren a las ideas de A. Vilanova y A. Gimeno profesores de (ESADE), de Barcelona, donde imparten una asignatura específica llamada La gestión de la empresa familiar, sobre un estudio de 206 empresas familiares de todos los sectores situadas sobre todo en Cataluña. Citado en S. ToBARRA (1997) «Propietario y gerente», El País, 19 de enero. Suplemento de Negocios. También hemos utilizado el trabajo publicado recientemente por el Servicio de Estudios la La Caixa en su colección de Estudios e Informe titulado La sucesión en la empresa familiar: aspectos organizativos y fiscales (1998).

(42) Se estima que en España el 71\% de las empresas que facturan más de 200 millones de pesetas son empresas de esta naturaleza. 
perdurabilidad de diversas formas (43). Entre otras pueden destacarse:

a) Su orientación hacia la autofinanciación, es decir, presentar balances con muchos fondos propios y pocas deudas bancarias. Los propietarios acostumbran a ponerse un sueldo y a repartir pocos dividendos (44). El inconveniente es que generalmente existe cierta confusión entre la caja de la empresa y la economía familiar.

b) Los recursos humanos y los de capital salen del mismo sitio. Se debe intentar mantener relaciones empresariales adecuadas por encima de los lazos familiares, que incluso pueden no ser de primer grado, sino que en las empresas antiguas llegan hasta familiares lejanos.

c) La idea del largo plazo se encuentra arraigada. Se piensa más en el futuro de la empresa porque está ligada a la familia, más allá del propio presente de las personas que la dirigen y que son sus actuales propietarios. Ello requirirá, entre otras cuestiones, clarificar los procesos decisorios entre los diversos ámbitos -consejo de administración, propietarios, directivos y familia-que previsiblemente se irán haciendo más complejos (45).

d) Tienen dificultades para sustituir al miembro de la familia que hace de líder en la empresa (46). Hasta los años sesenta, la dirección de la empresa le solía tocar al hijo mayor cuando el padre o el abuelo dejaban la primera línea, pero las cosas han cambiado, suele ser bastante normal que el miembro más preparado de la

(43) A. MAs-CoLELL señala la estructura de capital cerrada de las empresas familiares como un límite a sus posibilidades de crecimiento (A.MAS-CoLELL (1997) «El futuro de las pyme españolas», Expansión, 27 de mayo, p.30)

(44) El 44\% de las empresas optan por capitalizar los beneficios para tener fortaleza financiera suficiente y no pedir préstamos o reducirlos a la mínima expresión. Asimismo, tres cuarta partes de las empresas hacen las ampliaciones de capital sólo para la familia. Las estrategias están divididas a la hora de elegir entre la rentabilidad a corto y a largo plazo. El 53\% de las empresas dicen que tiene como objetivo la rentabilidad «sólo a largo plazo».

(45) El establecimiento de un «protocolo familiar» reviste así una especial importancia para asegurar la continuidad del espíritu empresarial, siempre y cuando éste no sea un conjunto de reglas rígidas que impidan el desarrollo futuro de la empresa. El empresario no deberá pretender «reinar después de muerto», sino, al contrario, establecer un sistema de valores que promueva el consenso entre los herederos en pro del beneficio conjunto.

(46) E1 72\% de las empresas consultadas admiten que tienen o han tenido dificultades para sustituir al líder familiar. Resulta interesante en este aspecto el trabajo de J.M. AmAT SALAS (1998) «La continuidad de la empresa familiar», Boletín AECA, n 47, pp.2-6. 
familia sea el que se haga cargo de la dirección general. Con todo, aún hay una asignatura pendiente: la llegada de las mujeres a la primera línea ejecutiva. Otra cuestión clave es el momento del relevo ya que muchos padres y abuelos se resisten a dejar las riendas del negocio, al considerarse imprescindibles. Conviene portanto desarrollar una planificación del proceso de sucesión y de preparación de los mismos (47). Muchas veces retirarse no supone tener que dejar la empresa. Al contrario, puede ser útil para la organización que el fundador permanezca en un segundo plano garantizando un proceso de transición más fluido y satisfactorio.

e) En la tercera generación (48) y en las siguientes suele haber choque entre las familias que están comprometidas con el proyecto y las que no lo están (49). Esta empresas crean a veces unos comités de familia para discutir los temas al margen de los órganos de dirección, es decir, el Consejo de Administración y los comités directivos.

Portanto, familia, sucesión, y relevo son cuestiones indisolublemente asociadas a la PYME que pretenda plantearse un horizonte más allá de la mera supervivencia.

(47) El primer paso en la planificación de la sucesión es la preparación de los sucesores, de modo que éstos alcancen una preparación humana y técnica coherente con el espíritu del emprendedor y las necesidades de un profesional de la dirección. La preparación de la sucesión se desarrolla en diversas fases. La remota, orientada a la inculcación de virtudes humanas de carácter general. La próxima, más vinculada a la preparación académica técnica. Y la inmediata, dedicada a los aspectos más particulares, que entraña la incorporación a la empresa familiar. Con todo, el proceso de preparación del sucesor puede no dar los resultados apetecidos y puede plantearse una sucesión por parte de los directivos no familiares.

(48)ElProfesor M. Gallo identifica las siguientes dificultades asociadas al paso de la segunda a la tercera generación: el distanciamiento familiar, la mayor dilución de la propiedad y, por tanto, desde el punto de vista de implicación en la actividad como de exigencias de rentabilidad sobre el capital. En cuanto a las orientaciones, este autor aboga por fortalecer el espíritu de empresa familiar al tiempo que se considera útil establecer métodos que permitan la desvinculación de la propiedad de los miembros familiares no interesados directamente en el proyecto.

(49) Vilanova y Gimeno quieren acabar con el mito de que los nietos suelen vender la empresa que construyó con esfuerzo el abuelo. «No es verdad ese refrán que dice que el abuelo crea la empresa, el hijo la hace crecer y el nieto o el bisnieto la vende. Hay bastantes empresas de tercera generación omás», señala Albert Gimeno. Un 13\%, según Gimeno y Vilanova, son empresarios de tercera generación o más, algo que no es fácil de alcanzar en el actual mundo competitivo y cambiante. Citado en S. ToBARRA (1997) «Propietario y gerente», El País, 19 de enero. Suplemento de Negocios. 


\section{c) Los condicionamientos exógenos en la dinámica empresarial}

Las instituciones públicas vienes desarrollando un papel activo de fomento de la actividad empresarial a través de PYME (50). Los organismos representativos de las PYME vienen solicitando insistentemente la adopción de nuevas medidas en distintos ámbitos -tributario, administrativo, laboral, seguridad social(51)-para potenciar el desarrollo de este tipo de experiencias. La OCDE (1997) considera en un estudio sobre la reforma de la empresa pública y el empresario la burocracia, la innovación y la financiación como factores limitantes para el dinamismo empresarial(52).

\section{c.1) La burocracia}

La lentitud de la organización pública en el reconocimiento y legitimación de la actividad empresarial presenta tres manifestaciones diferenciadas:

- Trabas burocráticas en la creación: Cada trámite exige un promedio de cuatro documentos distintos e implica a un mínimo de seis organismos diferentes, y el resultado de todo esto es que la empresa, antes de poder comenzar legalmente sus actividades, tiene que esperar entre diecinueve y veintiocho semanas. En Estados Unidos se necesita medio día paraestablecer una nueva empresa(53).En resumen,

(50) En España existe una Dirección General de Política de la Pequeña y Mediana Empresa. Para obtener información actualizada de las ayudas y otras informaciones relacionadas con el fomento de la PYME en España puede consultarse en internet la siguiente dirección: htpp:// www.mcx.es/pyme

(51)Estas reivindicaciones pueden sintetizarse en las siguientes: de orden tributario, con objeto de reforzar los recursos propios, la supervivencia de las empresas recientes y las inversiones en formación; en el entorno administrativo, para reducir las cargas administrativas y facilitar la información y asistencia técnica necesarias; en el marco laboral, para proseguir la reforma emprendida y hacer posible a las PYME la mejor adecuación de la plantilla a las necesidades cambiantes del mercado; en las cargas sociales, ya que estas actúan como un impuesto sobre el empleo, penalizando especialmente a las PYME por ser intensivas en mano de obra.

(52) Elaborado a partir de O. Martí (1998) «Los pecados de la burocracia», El País, 3 de mayo.

(53) La complejidad de la madeja administrativa fue evocada por el Financial Times en Abril de 1998, relatando la lista de instancias a la que puede que haya que presentar documentos. «Primero hay que proporcionar datos al ayuntamiento local y luego al registro industrial 
dice el informe, la creación de una nueva empresa es un proceso particularmente largo y complejo que obstaculiza el desarrollo de compañias más flexibles.

- Obstáculos para abandonar la actividad empresarial: Si poner en pie una empresa es difícil, cerrarla puede calificarse de «proceso complicado y oneroso» hasta el punto de que en España incluso el coste de un fracaso es disuasorio para un empresario y obstaculiza la asunción de riesgos de esta naturaleza.

- Incertidumbres en la protección del tráfico mercantil: La OCDE se refiere al sistema judicial «cuya eficacia habría que mejorar para garantizar un mayor respeto de las disposiciones contractuales».

\section{c.2) El ambiente de innovación}

Las oportunidades de innovación tecnológica son menos numerosas en España que en otros países. El número de investigadores por cada 10.000 trabajadores en Españaes de 30, la mitad que en Francia (54). Si en los Estados Unidos esos talentos básicos para la innovación están, en un 79\%, ligados a la empresa-un 70\% en el Japón, un 56\% en Alemania, un 45\% en Francia- en España el porcentaje es modesto: un $23 \%$. El resultado de todo ello es, afirma la OCDE, un tejido empresarial «cuya capacidad para generar o adaptar nuevas ideas es muy limitado». Conclusiones similares pueden obtenerse del coeficiente de inventiva calculado a partir del número de patentes registrado por cada 10.000 habitantes, en donde España sólo aparece por delante de Portugal y Turquía y muy lejos de suizos, australianos, suecos y alemanes.

del gobierno regional, al registro de la propiedad, a la oficina de la Seguridad social, al ministerio de Economía, al de Trabajo y puede que a las autoridades regionales que tengan competencias sobre Trabajo. Cada organismo puede requerir entre tres y diez documentos y cada uno de estos ha de presentarse porduplicado, triplicado o cuadriplicado». En resumen toda nueva empresa tiene que cumplir entre trece o catorce formalidades generales antes de iniciar su actividad - con formalidades suplementarias que se aplican en ciertos sectores». Si la fórmula escogida es una sociedad, entonces hay que contar con un mínimo de cinco trámites adicionales

(54) Pero ese dato en sí no sería alarmante -Italia tiene 33 investigadores por cada 10.000 trabajadores, Austria 34, Gran Bretaña 51, Japón 81- si no fuera porque la mayoría (un 60\%) dedica su actividad a la enseñanza universitaria. 


\section{c.3) Los problemas de financiación}

Entre las pequeñas y medianas empresas españolas, sólo un $47 \%$ de las entrevistadas en la Encuesta a Empresas Europeas percibe que tenía acceso suficiente a la financiación necesaria para sacar adelante sus proyectos en los tres próximos años, frente a una media europea del $55 \%$. Esta percepción de dificultad contrasta con un entorno en donde los tipos de interés son más bajos y existe una inflación controlada. El primer problema que se observaes que España sigue siendo «uno de los países en los que los términos medios de pago son más largos (73 días) dentro de la zona OCDE». Este retraso en el pago no queda compensado-la mitad de las empresas nunca aplican intereses a estos retrasos-. En ese sentido, es significativo que el nivel de desconfianza de las entidades bancarias o de prepotencia en las relaciones empresa grande-pequeña, las pequeñas empresas españolas se muestren las más puntuales en materia de pagos en el conjunto de Europa y las grandes sean también las que pagan con más retraso en el continente. En todo caso, pese a las mejoras en las condiciones crediticias, obtener financiación ha sido difícil y costoso para muchas empresas españolas (55). Posiblemente, según se desprende del informe de la OCDE, esta elevada percepción empresarial sobre las dificultades que les plantea la financiación en un entorno de tipo de interés alabaja se debaa lainclinación de labanca española al «racionamiento deloscréditos», a concentrar sus actividades en las empresas más asentadas y de mayor tamaño.

Una vez establecido el marco de los condicionantes internos y externos de la PYMEa nadie se les escapa el hecho de que algunas tienen éxito casi independientemente del tamaño. Surge entonces la pregunta: ¿qué orientaciones básicas adoptan ese colectivo de PYME que se desenvuelve con éxito aprovechando sus ventajas a pesar de los condicionamientos de su dimensión?

\section{Factores para la excelencia empresarial en la PYME}

La pequeña dimensión no será sinónimo de eficacia y los puestos de trabajo seguirán siendo insuficientes y precarios mientras no se pongan al servicio de

(55) Durante 1997, el 43\% de las compañías entrevistadas en la Encuesta a Empresas Europeas citaba el coste de la financiación como una restricción a corto plazo para la expansión de sus negocios, mientras que un $32 \%$ la citaba como una restricción a largo plazo. 
unidades productivas capaces de generar bienes y servicios con calidad y precios competitivos, dentro del contexto que exigen las nuevas circunstancias. Esta proposición no es sólo un deseo sino una realidad para determinadas PYME-como las mittelstand alemanas (56) - que son capaces de desarrollarse utilizando y combinando sus recursos y oportunidades de forma estratégica. A continuación presentamos algunas de estás lineas básicas orientativas de pequeñas y medianas empresas de éxito entresacadas de diversos estudios (Cliford y Cavanagh, 1989; Muzyka y Churchill, 1996; Breuninger y Muzyka, 1996):

\section{a) Mantener el impulso inicial: orientados hacia las oportunidades}

¿Por qué tantas organizaciones emprendedoras pierden la capacidad para identificar y perseguir las nuevas oportunidades que se presentan?En primer lugar, tienden a centrarse demasiado en las actividades que les dieron el éxito, descuidando el proceso organizativo, que desempeña un papel relevante en su resultado excelente. En segundo lugar, intentan en vano mantener lo que se tiene mediante la implantación de un excesivo número de normas, sistemas y controles.

En el caso de las mittelstand alemanas la orientación estratégica estaba dirigida básicamente a la identificación y el aprovechamiento de oportunidades, asegurar la renovación de las líneas de producto y las actividades de la empresa. Tradicionalmente, muchas de estas empresas han producido y vendido productos basados exclusivamente en su tecnología y en sus conocimientos especializados. La actitud predominante sería: si creemos que es mejor, el mercado tiene que aceptarlo porque incorpora nuestra mejora tecnológica (57). Sin embargo las PYME de éxito parecen tener un modelo iterativo de crear e introducir nuevos productos y servicios. Este proceso comprende la búsqueda de una combinación

(56) La mittelstand constituye un tipo de empresa característica de Alemania Occidental perteneciente al sector de las pequeñas y medianas. Tres cuartas partes de ellas son empresas familiares y un elevado porcentaje está constituido por empresas manufactureras. Muchas de ellas se crearon incluso a principios de siglo, mientras que otras se fundaron después de la Segunda Guerra Mundial.

(57) En el ámbito de las PYME de alta tecnología existe un estudio de casos de 18 empresas que confirma alguna de las propuestas que hacemos. Ver I. MARCH CHORDÁ (1998), «Modelización empírica del comportamiento PYMEs high-tech innovadoras», Revista de Economíay Empresa, $\mathrm{n}^{\circ} 32$, Volumen XII, $1^{\circ}$ cuatrimestre, pp.43-64. 
de necesidades reales de los clientes y las competencias que la empresa puede aportar para crear valor para ambas partes. Una consecuencia de este enfoque es que el desarrollo de productos se realiza mediante contactos sucesivos con los clientes (58). Esto, a su vez, conduce a las empresas a buscar nichos de mercado y a crecer mediante la explotación de sucesivas oportunidades de nicho. Han adquirido una mejor comprensión de la necesidad de aprovechar oportunidades para renovarse y crecer, por un lado, y una conciencia interna más generalizada de la necesidad de centrarse en las oportunidades a todos los niveles, por otro.

\section{b) Horizontalidad frente a verticalidad}

Las empresas investigadas (Breuninger y Muzyka,1996) trabajan mediante equipos y grupos de proyecto, y los nuevos problemas o desafíos se abordan horizontalmente más que verticalmente. Los equipos integrados por miembros de diferentes departamentos son capaces de cooperar y procesar las innovaciones. Ladirección de la empresa se esfuerza agresivamente por mejorar la comunicación y la transferencia de conocimientos llegándose a encargar a los diseñadores o los directores de producto - que casi nunca ven a un cliente- la realización de investigaciones de mercado entre los usuarios finales. La principal mejora radica, sin embargo, en la creación de nuevos productos y servicios mediante un enfoque cooperativo que utiliza una tecnología muy diversa. Las personas han dejado de sentirse seguras u orgullosas de sus conocimientos técnicos a nivel individual; ahora tienen que aplicarlo con el resto de la empresa para crear valor.

\section{c) Instalados en el cambio}

El concepto de cambio y de adaptación permanente también se encuentra profundamente arraigado en estas organizaciones. La transformación fluida (en función de las necesidades pero sin mucha planificación) se ha convertido en un modo de vida. La incidencia de este proceso en el personal parece haberse minimizado eludiendo las reducciones de plantilla y poniendo el énfasis en la

(58) Puede verse el caso de la empresa Thermos y el proceso de creación y diseño de una barbacoa eléctrica (A.C. Morales Gutiérrez, J.A. Ariza Montes (1995)Las organizaciones. Casos de análisis organizativo. Publicaciones ETEA, pp.89-96) 
reorientación y la reconversión profesional. En varios casos, estas organizaciones han hecho gala de un enfoque del desarrollo de oportunidades y productos innovadores substancialmente más intertecnológicoe interfuncional (combinando distintas competencias y tecnologías con el fin de satisfacer las necesidades del cliente). Muchos de los productos desarrollados han surgido de este proceso de compartir los conocimientos tecnológicos, y no de la búsqueda de mejores soluciones para una tecnología dada.

\section{d) Centrados en la eficacia}

Convencidos de los peligros de una excesiva certidumbre y estabilidad ¿cuál es el anclaje cuando uno se instala en el cambio? La excelencia empresarial precisa una actitud de comprensión de la necesidad de alcanzar cotas elevadas de eficacia que se encuentre generalizada y extendida por toda la organización. En la organizaciones investigadas la eficacia adquirióuna importancia primordial a nivel de toda la organización, de los equipos y de las personas, y el cumplimiento de los objetivos de la empresa pasó a ser objeto de una comunicación más abierta. En estas organizaciones aumentó el grado de responsabilidad y de conciencia sobre la eficacia general de la organización. En las organizaciones innovadoras seleccionadas, prácticamente todos los empleados comprendían los objetivos no sólo de su propia unidad, sino del conjunto de la empresa. Sus direcciones habían reconocido que si la gente no sabe a dónde va, es muy probable que escojan al azar cualquier camino.

\section{e) Gestión de información equivale a gestión de poder de mercado}

Un pilar fundamental de los logros alcanzados por estas organizaciones ha sido la mejora de los conocimientos y de la gestión de la información. Esta gestión de la información no sólo comprende las soluciones de hardware, sino también una mejora de los procesos de recogida, análisis y difusión de los conocimientos dentro de la organización. En estas organizaciones, la información está ampliamente disponible. Esto ha surtido también sus efectos sobre los mecanismos de control. La amplia disponibilidad de información sobre los resultados y la reacción de los mercados ha hecho que el control operativo sea más sutil y difuso. Debido a la mayor visibilidad y transparencia existentes, cada persona procura controlar sus 
propias acciones. En la mayoría de los casos, el disponer de hechos y no sólo de opiniones o convicciones ha contribuido a mejorar la toma de decisiones, actuar con mayor rapidez y promover la cooperación entre distintas tecnologías y funciones.

\section{d) Creadores de valor: lo que no está en el proceso no está en el producto}

Las organizaciones seleccionadas han adoptado medidas para racionalizar su cadena de valor, centrándose en las actividades claves para desarrollar el potencial de crecimiento de la empresa y su rentabilidad en el futuro. Se han planteado activamente la cuestión ¿dónde añadimos valor? Como consecuencia de esta reflexión las empresas establecieron fuertes relaciones con sus proveedores, especialmente a nivel internacional. Pese a su tamaño, estas empresas han intentado reducir sus costes trasladando al menos una parte de su producción fuera de las regiones con unos costes más elevados, manteniendo la calidad de la producción mediante los controles apropiados. Las organizaciones miden el rendimiento de un modo que supone una visión más equilibrada del éxito al contemplar tanto la eficiencia de las operaciones -costes-como su eficacia- ¿es posible alcanzarobjetivos desafiantes?-. Esto les hace preguntarse hasta qué punto están sacrificando sus posibilidades de crecimiento o renovación en aras de los beneficios a corto plazo.

\section{f) Al éxito por la cooperación}

La globalización de los mercados exige superar individualismos improductivos, para redimensionar la estructura empresarial a través de estrategias que refuercen el poder de negociación de las PYME en su relación con otras empresas, con las Administraciones Públicas o con las entidades financieras. En su política de expansión, las empresas han seguido estrategias de fusión, adquisición y cooperación, a través de las cuales se ha producido una concentración en busca de economías de escala, de posicionamiento en un mercado o de ambas cosas a la vez: la cooperación empresarial, a diferencia de las fusiones y adquisiciones, no genera una relación de subordinación entre empresas; éstas conservan plena autonomía operativa en aquellas parcelas de actividad en que no se ha acordado la cooperación. Sin embargo, proporciona la oportunidad de racionalizar gastos, 
compartir riesgos y establecer relaciones comerciales más equilibradas con otros agentes competitivos. La cooperación empresarial abre una amplia gama de posiblidades de actuación en función de las necesidades e imaginación de los empresarios para servirse de fórmulas en distintos ámbitos como el:

- jurídico (con forma jurídica (59) o simple acuerdo entre empresas);

- comercial (consorcios, cadenas de compras...) (60);

- tecnológico (acciones colectivas, subcontratación, calidad concertada...);

- financiero (sociedades conjuntas, sociedades de garantía recíproca y sociedades de riesgo);

- formativo (diagnóstico de necesidades, planes conjuntos de formación, planes sectoriales...) (61).

Es fundamental la mentalización de los empresarios para realizar el cambio que supone el contemplar como posibles colaboradores a sus competidores. Sería deseable que aumentase en número y prospere la confianza entre cooperantes nacionales y extiendan su actividad hacia objetivos de contenido tecnológico, con vistas a una mayor competitividad de los bienes y servicios españoles dentro y fuera de nuestras fronteras.

Por tanto para mantener vivo el espíritu empresarial se precisa: concentrar a las

(59) Las figuras jurídicas más utilizadas para la cooperación empresarial son las Uniones Temporales de Empresas (UTE)- reguladas por la Ley de Régimen Fiscal de las Agrupaciones y Uniones Temporales de Empresas, de 26 de mayo de 1982- y las Agrupaciones de Interés Económico (AIE). La UTE pueden ser definidas como un sistema de colaboración entre empresario por tiempo cierto, determinado o indeterminado, para el desarrollo de una obra, servicio o suministro. Generalmente se procede a realizar la misma actividad que los asociados y no una simple actividad auxiliar. La AIE es una sociedad que se crea por los socios para que les apoye en la actividad propia de ellos y no buscando obtener un beneficio aunque si pueden tenerlo. Sobre este tema puede verse M.A. BERGAS JANÉ (1998) «Fórmulas jurídicas para la cooperación empresarial», Estrategia Financiera, n¹38, Marzo, pp. 30-33.

(60) Por citar algunos datos: en 1993 se formalizaron 154 acuerdos entre empresas, con una subvención media por proyecto de 2.600 .000 pesetas. Dos terceras partes tenían ámbito transnacional y el $65 \%$ estaban destinados a objetivos comerciales. En este ámbito resulta muy interesante el estudio de E. GARCíA CANAL (1992) «La cooperación interempresarial en España: características de los acuerdos suscritos entre 1986-1989», Economía Industrial, Julio-Agosto, pp.113-122.

(61) En este sentido FORCEM posibilita una serie de oportunidades para las PYMEque están siendoinsuficientemente aprovechadas. 
personas en las oportunidades, el crecimiento y la renovación; abrir los circuitos informativos de tal forma en que no se conviertan en una fuente de poder individual de los directivos; incentivar a las personas para que compartan los conocimientos, la tecnología y las capacidades para perseguir y explotar oportunidades; asegurarse de que las personas y los equipos cuentan con la formación y la preparación necesarias para poder apoyarlos en sus actividades; renunciar a una parte del deseo de adoptar todas las decisiones de forma centralizada; y considerar la cooperación como una estrategia posible y razonable.

\section{Consideraciones finales}

El fenómeno de las PYME ha sido tratado, quizás por su aparente simplicidad, con demasiados estereotipos que no responden en muchos casos a su realidad cotidiana. Los cambios acontecidos en las estructuras económicas de los países occidentales han puesto de manifiesto la necesidad de contar con estos agentes económicos potencialmente vulnerables pero también dinamizadores de la actividad económica. Se constituyen, de esta forma, en protagonistas de un desarrollo económico más equilibrado, más participativo y más arraigado.

La vitalidad de la economía depende, cada vez más, de la vitalidad de las PYME. Su competitividad condiciona el crecimiento y desarrollo de todo el sistema, al tiempo que se apoya en elementos de carácter social, educacional, tecnológico, económico y normativo que es necesario identificar y potenciar para conformar un tejido empresarial sano.

El escenario futuro que se le presenta a las PYME muestra ciertas oportunidades. La oferta se diversifica y se orienta de forma más individualizada hacia los consumidores, lo que ha obligado a una fragmentación del mercado y, en ciertas ocasiones, a una producción de bienes y servicios en series más cortas, susceptible de ser realizada por empresas de pequeña dimensión. Este proceso de cambio acelerado ha obligado a las empresas a modificar las estrategias competitivas, orientándolas hacia formas menos rígidas de actuación, a través de la descentralización de funciones y servicios, de nuevas formas de subcontratación que proporcionan mayores oportunidades de negocio a las pequeñas y medianas empresas. Movilidad y flexibilidad constituyen, por tanto, claves de progreso y de estabilidad social en un entorno de creciente presión sobre las unidades productivas como consecuencia de unos mercados cada vez más complejos y diversificados. 
Las grandes empresas se reestructuran a velocidades vertiginosas y mientras tanto las PYME siguen siendo un indicador de la capacidad de una sociedad que busca la solución de sus problemas, y no espera a que las soluciones vengan desde otras instancias. Al margen de las responsabilidades de los agentes sociales -organizaciones representativas y administración-en aras de establecer tratamientos específicos, reducir las barreras para la creación de empresas y a la defensa de la competencia, y promover mecanismos de fomento adecuado, las PYME deberán optar por mantener su caracter innovador, centrarse en la calidad y en la generación de valor, potenciar el desarrollo de sus recursos humanos y no obviar las posibilidades de acción conjunta que el nuevo entorno empresarial parece ofrecerle y exigirle.

\section{Bibliografía}

Amat Salas, J.M. (1998) «La continuidad de la empresa familiar», Boletín AECA, $\mathrm{n}^{\circ} 47, \mathrm{pp} .2-6$.

BERGAS JANÉ, M.A. (1998) «Fórmulas jurídicas para la cooperación empresarial», Estrategia Financiera, nº138, Marzo, pp. 30-33.

Breuninger, H. Daniel, F. MuzyKa (1996) «La reinvención de la Mittlestand», Nuevas ideas de Gestión Empresarial, Expansión, pp.9-11.

CES (1995), La fuerza emergente de la PYME en Informe sobre la situación española, Fundación SM.

Cliford, D.K., Cavanagh, R. E. (1989) Estrategias de éxito para la pequeña y mediana empresa, Folio, Barcelona.

Chaganti, R (1987) «Small Business Strategies in Different Industry Growth Evironments». Journal of Small Business Management, Vol. 25, № 3, Jul. 1987, pp. 61-68.

Churchill, N. (1996), «Las seis fases claves del crecimiento de la empresa», Nuevas ideas de gestión empresarial, $\mathrm{n}^{\circ} 8$ Expansión, pp.2-5.

ERnst \& Young, (1989) La PYME española y el Mercado Unico. IMPI.Colección Estudios.

Evans, D.S.; Leighton, L. S. (1989) «Some Empirical Aspects of Entrepreneurship». American Economic Review, Vol. 79. № 3, Jun., pp. 519-535.

GARCía CANAL, E.(1992) «La cooperación interempresarial en España: caracte- 
rísticas de los acuerdos suscritos entre 1986-1989», Economía Industrial, Julio-Agosto, pp.113-122.

Harrison, B. (1994) «El mito de las pequeñas empresas», Harvard Deusto Business Review, $\mathrm{n}^{\circ}$ 5, pp.30-39.

Harrison, B. (1997) La empresa que viene, Paidós, Barcelona.

Hermosilla, A., SolÁ, J. (1989) Cooperación entre empresas, IMPI, Colección Estudios. Madrid.

Haswell, S.; Holmes, S. (1989) «Estimating the Small Business Failure Rate: A Reappraisal». Journal of Small Business Management, Vol. 27, Nº 3, Jul., pp. $68-74$.

Levié, J y Hay, M. (1996) «Más allá de la cultura de cocina», Nuevas ideas de Gestión Empresarial, Expansión, no 8, pp.4-6.

MARCH CHORDÁ, I (1998) «Modelización empírica del comportamiento PYMEs high-tech innovadoras», Revista de Economía y Empresa, n 32, Volumen XII, $1^{\circ}$ cuatrimestre, pp.43-64.

Martí, O. (1998) «Los pecados de la burocracia», El País, 3 de mayo.

Mas-Colell, A. (1997) «El futuro de las pyme española», Expansión, 27 de mayo, p.30)

MuzyKa, D.F. (1996), «Cómo identificar oportunidades». Nuevas ideas de Gestión Empresarial, Expansión, n⿳0 2,pp. 4-6.

Muzyka, D.F. y Neil Churchill (1996) «Cómo fomentar el espíritu de empresa. Nuevas ideas de gestión empresarial», Expansión, nº 9, pp.8-11.

Preisendorfer, P.; Voss, T.(1990) «Organizational Mortality of Small Firms: The Effects of Entepreneurial Age and Human Capital».Organization Studies, Vol. $11, N^{\circ} 1,1990$, pp. 107-129.

Roure, J. (1996) «Diez mitos sobre los empresarios», Nuevas ideas de Gestión Empresarial, Expansión, p.7.

Sainte-Marie, G. De (1995) Dirigir una PYME, Paidós. Barcelona

Tobarra, S. (1997) «Propietario y gerente», El País, 19 de enero. Suplemento de Negocios 